\title{
Provocative testing for low-risk chest pain patients, must we continue?
}

\author{
James Booth, $M D,{ }^{a}$ and J. Jeremy Thomas, $M D^{a}$ \\ a Emergency Medicine, UAB Health System, Birmingham, AL
}

Received Jan 16, 2018; accepted Jan 16, 2018

doi: $10.1007 / \mathrm{s} 12350-018-1202-2$

\section{See related article, pp. 1642-1646}

Between 6 and 10 million patients are brought to US emergency departments with chest pain annually, accounting for 5 to $10 \%$ of all ED visits. ${ }^{1,2}$ A subset of these patients will have acute coronary syndrome (ACS) including an acute myocardial infarction (MI). Missing an acute $\mathrm{MI}$ is a significant risk of morbidity for patients and risk of litigation for emergency physicians. While a historical miss rate of $2 \%$ to $4 \%$ is commonly quoted, more recent data suggest that current diagnostic strategies reduce this miss rate below $1 \% .^{3-5}$ While this reduction in missed MIs is desirable, it comes at the cost of increased rates of hospital admissions for chest pain "rule outs" and additional diagnostic tests, accruing more than $\$ 3$ billion in annual hospital costs in the US and subjecting many patients to testing that may not be necessary. ${ }^{6,7}$

A subset of patients with "low-risk" or "moderaterisk" chest pain, as determined by risk assessment tools or physician clinical judgment, are often admitted to hospital observation units and subjected to additional non-invasive cardiac testing, such as a stress test or coronary computed tomographic angiogram (CCTA). This practice is supported by the 2014 ACC/AHA guidelines that recommend non-invasive cardiac testing within 72 hours of presentation in patients with negative cardiac markers and non-ischemic ECGs. ${ }^{8}$ In general, patients with negative stress tests or negative CCTAs are discharged home while patients with positive tests have

\footnotetext{
Reprint requests: J. Jeremy Thomas, MD, UAB Health System, Birmingham, AL; jjthomas@uabmc.edu

J Nucl Cardiol 2019;26:1647-9.

$1071-3581 / \$ 34.00$

Copyright (C) 2018 American Society of Nuclear Cardiology.
}

subsequent cardiology consultations to determine next steps including possible invasive cardiac catheterization.

The HEART score is a risk stratification tool used with increasing popularity in the emergency department (ED) setting for patients with chest pain. The score has been shown to reliably stratify patients with possible ACS into low, moderate, and high-risk categories based on 5 elements (history, ECG, age, risk factors, and troponin). Patients with HEART scores less than 4 and negative troponins may be discharged from the emergency department with a low $<1 \%$ rate of major adverse cardiac event (MACE) in the 90 days following ED evaluation. The reliability, ease of use, and efficiency of the HEART score has led to an increasing number of hospitals integrating the score into standardized chest pain protocols for their emergency departments. The use of the HEART score has been shown to decrease the number of patients admitted to hospitals for chest pain and thereby subsequently decrease the utilization of non-invasive cardiac testing, such as stress tests, to further risk stratify patients.

In this issue of the Journal, Krishnan et $\mathrm{al}^{9}$ publish results from a retrospective analysis of 292 emergency department patients with chest pain. These patients were deemed low risk and subsequently placed in an observation unit to receive additional non-invasive cardiac testing. $69 \%$ of the patients underwent pharmacologic or exercise stress tests with myocardial perfusion imaging (MPI) while the remainder underwent exercise stress tests without imaging. 33 patients $(11.3 \%)$ had positive stress tests for ischemia. $50 \%$ of these 33 patients had a prior history of $\mathrm{MI}$, percutaneous coronary intervention (PCI), or coronary artery bypass graph (CABG). Of these patients with positive stress tests, 12 underwent subsequent diagnostic cardiac catheterization and only 4 of these patients ( $1.4 \%$ of the total 292 patients) had revascularization with possible mortality benefit.

While the results published by Krishnan et al are retrospective and limited to a single center, they add to a growing body of recent literature that suggests that 
cardiac stress tests are overutilized in emergency department patients admitted (or even discharged with expedited provocative testing as an outpatient) for lowrisk chest pain. ${ }^{7,10-13}$ As one example, a recent large retrospective analysis by Sandhu et al looked at an insurance claims database of over 900,000 ED chest pain patients with initial negative workups. The results showed no significant reduction in subsequent admissions for acute MIs in patients who underwent noninvasive cardiac testing for further risk stratification. Another recent study by Reinhardt et $\mathrm{al}^{13}$ looked at 1,000 ED chest pain patients across 9 hospitals with initial negative workups for ischemia who were randomized to non-invasive cardiac testing vs clinical evaluation alone. The results showed that non-invasive testing led to longer lengths of stay (LOS), greater costs, more downstream testing, and more radiation exposure without improvement in clinical outcomes.

In addition to the majority of stress tests being negative for ischemia, the data from Krishnan et al also show that the majority of studied patients with positive stress tests did not undergo revascularization therapy. 21 of the 33 patients $(63.6 \%)$ with positive stress tests were discharged without cardiac catheterization during their hospital stay. 13 were directly discharged from the observation unit with cardiology follow-up and 8 patients underwent further non-invasive tests such as CCTA, repeat stress test, or transthoracic echocardiogram (TTE). The decision to proceed with cardiac catheterization was made by the consulting cardiologist without a standardized decision rule but mostly based upon the size of the ischemic area noted on the MPI.

An unfortunate limitation to the Krishnan et al analysis is the lack of standardization for determining the patient's risk of ACS. The emergency physicians at the time deemed the patients to be "low-risk" yet not low-enough-risk to be sent home directly from the emergency department without further non-invasive cardiac testing. Use of the HEART score could have potentially resulted in a percentage of the 292 patients being discharged directly from the emergency department without undergoing further stress testing. Unfortunately, limitations in the patient documentation prevent a retrospective application of the HEART to this study population.

The general approach to emergency department patients with chest pain has been evolving over recent years with the increasing use of the HEART score and high-sensitivity troponins. Despite the 2014 ACC/AHA guidelines, more "low-risk" patients are being discharged directly from emergency departments without undergoing further non-invasive cardiac testing (in the inpatient, observation, or expedited outpatient setting). The analysis by Krishnan et al and other similar studies now raise questions regarding the best approach to patients who are deemed "moderate-risk" (HEART scores of 4-6) or "high-risk" (HEART scores $>6$ ) and whether these patient populations benefit from undergoing non-invasive cardiac testing in the acute setting. As Krishnan et al identify in their results, $50 \%$ of the patients who had positive stress tests had prior histories of CAD. Future directions for research may include a prospective randomized clinical trial of early non-invasive testing and the utilization of the HEART score and prior history of CAD to help guide a more targeted higher-yield patient population.

In the setting of ongoing national health care reform, universal emphasis on healthcare cost savings and quality improvement, as well as the desire to standardize practice based on evidence-based medicine- the current universal practice of liberal provocative testing will likely come under intense scrutiny in the near future. Provocative testing is time consuming, expensive, and often difficult to coordinate rapidly as an outpatient from the ED setting. These issues often lead to unnecessary observation stays and admissions to the hospital that have shown no benefit to these low-risk patients, but create significant cost and resource utilization. With the growing body of evidence showing a lack of benefit (in mortality or MACE) to low-risk ED chest pain patients that undergo provocative testing in the setting of a negative ED evaluation for ACS, we anticipate (and hope) that future revisions of the ACC/ AHA guidelines will soften the recommendation for provocative testing within 72 hours of ED evaluation for this patient population.

\section{Disclosure}

James Booth and J. Jeremy Thomas declare that they have no conflict of interest to disclose.

\section{References}

1. Bhuiya FA, Pitts SR, McCaig LF. Emergency department visits for chest pain and abdominal pain: United States, 1999-2008. NCHS Data Brief 2010;43:1-8.

2. Owens PL, Barrett ML, Gibson TB, Andrews RM, Weinick RM, Mutter RL. Emergency department care in the United States: A profile of national data sources. Ann Emerg Med 2010;56:150-65.

3. Pope JH, Aufderheide TP, Ruthazer R, Woolard RH, Feldman JA, Beshansky JR, et al. Missed diagnoses of acute cardiac ischemia in the emergency department. N Engl J Med 2000;342:1163-70.

4. Weinstock MB, Weingart S, Orth F, VanFossen D, Kaide C, Anderson $\mathrm{J}$, et al. Risk for clinically relevant adverse cardiac events in patients with chest pain at hospital admission. JAMA Intern Med 2015;175:1207-12.

5. Than M, Herbert M, Flaws D, Cullen L, Hess E, Hollander JE, et al. What is an acceptable risk of major adverse cardiac event in 
chest pain patients soon after discharge from the emergency department? A clinical survey. Int J Cardiol 2013;166:752-4.

6. Sabbatini AK, Nallamothu BK, Kocher KE. Reducing variation in hospital admissions from the emergency department for lowmortality conditions may produce savings. Health Aff (Millwood) 2014;33:1655-63.

7. Sandhu AT, Heidenreich PA, Bhattacharya J, Bundorf MK. Cardiovascular testing and clinical outcomes in Emergency Department Patients with chest pain. JAMA Intern Med 2017. https://doi.org/10.1001/jamainternmed.2017.2432.

8. Amsterdam EA, Wenger NK, Brindis RG, Casey DE, Ganiats TG, Holmes DR, et al. ACC/AHA Task Force Members. 2014 AHA/ ACC guideline for the management of patients with non-ST-elevation acute coronary syndromes: A report of the American College of Cardiology/American Heart Association Task Force on Practice Guidelines. Circulation 2014;130:e344-426.

9. Krishnan S, Venn R, Blumenthal D, Bhambhani V, Gewirtz H, Weiner RB, et al. Utilization of stress testing for low-risk patients with chest discomfort in the emergency department. J Nucl Cardiol 2018. https://doi.org/10.1007/s12350-017-1172-9.
10. Hermann LK, Newman DH, Pleasant WA, Rojanasarntikul D, Lakoff D, Goldberg SA, et al. Yield of Routine Provocative Cardiac Testing Among Patients in an Emergency DepartmentBased Chest Pain Unit. JAMA Intern Med 2013;173:1128-33.

11. Safavi KC, Li SX, Dharmarajan K, Venkatesh AK, Strait KM, Lin $\mathrm{H}$, et al. Hospital variation in the use of noninvasive cardiac imaging and its association with downstream testing, interventions, and outcomes. JAMA Intern Med 2014;174:546-53.

12. Foy AJ, Liu G, Davidson WR Jr, Sciamanna C, Leslie DL. Comparative effectiveness of diagnostic testing strategies in emergency department patients with chest pain: An analysis of downstream testing, interventions, and outcomes. JAMA Intern Med 2015;175:428-36.

13. Reinhardt SW, Lin C-J, Novak E, Brown DL, et al. Noninvasive cardiac testing versus clinical evaluation alone in acute chest pain: A secondary analysis of the ROMICAT-II randomized clinical trial. JAMA Intern Med 2017. https://doi.org/10.1001/jamainter nmed.2017.7360 